\title{
Artificial intelligence techniques for fault assessment in laminated composite structure: a review
}

\author{
Sidharth Patro ${ }^{l}$, Trupti Ranjan Mahapatra ${ }^{l *}$, Sushmita Dash $^{2}$ and Vikram Kishore Murty ${ }^{3}$ \\ ${ }^{1}$ Department of Production Engineering, Veer Surendra Sai University of Technology, Burla, Odisha, India \\ ${ }^{2}$ Department of Mechanical Engineering, Gandhi Institute for Technological Advancement, Bhubaneswar, Odisha, India \\ ${ }^{3}$ AIML Architect, Bengaluru, Karnataka, India
}

\begin{abstract}
There is a continuous quest in the research community for superior and more accurate methodology for fault diagnosis and condition monitoring of diverse composite structure. This is because, these structures suffer from various nonlinear mode of failures while in service those are recognised as delamination, voids, matrix crack etc. Early detection of failures is what the most research mainly aims at. In this regard, the implementation of Artificial Intelligence (AI) techniques has been proved to be a versatile method for damage assessment. The collective inevitable use of composite materials in various highperformance engineering industries requires preliminary testing (detection, location, and quantification) for damage to these materials in order to improve their integrity and order. The present paper aims to bring out a concise review on various methodologies employed for damage/fault detection in composite materials with a special emphasis on supervised and unsupervised machine learning techniques. The major observations are outlined with an objective to put forward a broad perspective of the state of art related to laminated composite structural heath monitoring.
\end{abstract}

\section{Introduction}

Composite materials are types of substances produced by combining two or more different chemical or physical ingredients in a visible level, without chemical reactions or melting, for instance as the final materials carrying the qualities of their properties. Presently, by reinforcing matrix materials composite materials are produced with good modulus fibers, good strength, flacks, or particles. The common reinforcing materials such as graphite, silicon carbide, boron and the matrix materials could be metal (Al, Ti), polymer (polyimides, epoxies), metal ( $\mathrm{Ti}$, $\mathrm{Al}$ ) or ceramic. Structural material formed by gathering stacks of composite materials produce laminated composite that are fibrous in nature to obtain engineering properties like in plane stiffness, coefficient of thermal expansion and bending stiffness Because of their amazing properties (e.g. high fatigue life, durability and optimal strength,), composite materials substitute common elements in different industries, such as aviation and aerospace, ocean, wind power, and automobiles [1]. Composite materials because of their heterogenous nature are prone to various complex types of damage. For example, interlaminar porosity, disbonding, fiber misalignment, matrix crack and core disbonding [2]. The damages may occur in the composite materials to the manufacturing process or during their operating life and consequently leads to the catastrophe failure in composite structure. The commencement and development of damage to the composite structures is in accordance with a complicated pattern and cannot be inspected by vision testing and generalised ways. This is due to the complex and hidden nature of the damage for example matrix crack, delamination. Therefore, judging of composite structures for the quantification, detection, and damage localisation is of vital importance for timid and authentic use of composite materials in practical implications.

Intelligent materials are those which reciprocate to external factors such as temperature, stress, electric field, magnetic field, humidity, $\mathrm{pH}$, etc. by modifying peripheral structures in a controlled manner. For instance, piping devices generate a type of machine where an electric field is used to generate electricity costs with regards to the flexibility of the equipment. Intelligent structures are those which are formed by combination of intelligent objects (facial or embedded, continuous or discrete) with structures through an actuator and sensor for making them intelligent. These structures are able to detect and modify the stable and dynamic response, and to continuously monitor the existence, complexity, and position of known damage from sensory details.[3]-[6] Applying sophisticated objects, a numerous of strategies are developed over the past two decades to assess the damage of intelligent components, such as wave-guided strategies [8], wave-based thinking strategies [7], [8], peri 
dynamics strategies based [9], and strategies based on vibration, among others.

According to Giurgiu Tiu [10], monitoring of structural health can be changed in two main ways: (a) idle SHM; and (b) applicable SHM. Passive SHM relies heavily on using multiple operating computers and determines the state of health of the structure from these variables. For example, one might see aircraft flight parameters (airspeed, air turbulence, factor, vibration levels, pressure in critical areas, etc.) and then use aircraft design algorithms to determine how much useful aircraft life has been used and is expected to leave how much. Passive SHM is useful, but it fails to deal directly with the crux of the problem, that is, it does not directly check whether the building is damaged or not. In contrast, the effective SHM is responsible for accurately measuring the health status of a building by trying to determine the presence and magnitude of structural damage. In this case, the SHM method is similar to the non-destructive test (NDE) method, only when the active SHM takes it one step further: the active SHM attempts to create permanent damage sensors in the building and monitoring mechanisms can provide bulletins health requirements. Generally Structural health monitoring (SHM) is divided into two ways: (a) passive SHM which considers the operational parameters of the system and draws the inference from it accessing the structural health of the system. From passive SHM one could monitor the overall remaining useful life of the system but could not totally access the structure damage which remains a major concern in determining the fault diagnosis by passive system. But (b) active system takes the extent of damage of structures. It is like non-destructive evaluation (NDT) methodologies as it takes the critical factors into consideration. So, the focal point will be on the active systems in this paper. Copious number of methods have been researched till now on active system.

In recent times, machine learning has emerged as smart tools for combining, retracting, and separating the elements of prejudice by illustrating the boundaries of complicated decisions on hyperplanes [11]-[14]. However, because of the prior discussion, it is impossible to define a large set of discriminatory factors which may be used to distinguish between all types of injury mechanisms (matrix crack, delamination, voids fiber fracture, etc.) in integrated structures. Also at the same time point, measure, and replace certain kinds of damage along with the identical set of discriminatory features. Additionally, one type of machine learning strategy can effectively produce damage but fail for others. Therefore, the purpose of this review article is to provide a comprehensive review of the sensitivity of the damage.

Rytter has embarked on a program to divide the longstanding state system that has been largely acquired by the clique responsible for the acquisition of damages and SHM. Using these lines, the nature of the damage is explained by answering the following questions (Sohn et al. (2003)): [15]

1. Pre-existence of Damage

2. Damage Detection on surfaces

3. Damage kinds

4. How the damage can affect the material
5. Is the damage low the remaining useful life (RUL) By identifying the type of injury and the extent we need pre-ethical views of the structure, with the predictable methods of failures of future assessment information and integration, which are often done using analytical models. Taking a performance monitoring case where we take the modal parameters of a damaged sample and compare it to the sample of its non-damaged structure. This is called global diagnosis. When damage is detected, we use a structural model in the damaged condition to assess the location of the damage. This is called Local diagnosis.

Availing the insightful materials, several approaches have been evolved in the last two decagon for the damage evolution of smart composite structures, just like guided wave-based methodology, wave field imaging-based methodology, peri dynamics-based methodology, and structural vibration-based methodology.

The main body of the article is dedicated to the review of important aspects of damage associated with several kinds of damage to integrated structures, as well as intelligent algorithms of types related to those features. Nevertheless, it will be beneficial to consider the aspects of machine learning.

Machine learning is a scientific area of science that finds the evolution and examination of mathematical \& statistical algorithms that can build effective relationships between numbers based on known knowledge and known laws.

Machine learning generally divided into three categories:

- Supervised Machine Learning

- Unsupervised Machine Learning

- Reinforcement Learning

As the name suggests Supervised learning has the existence of a supervisor which teaches it. If the data is labelled well, we could apply supervised machine learning to teach or train the machine. This means we have some data already linked with the right answer. This acts as the training data so that when machine is given a new set of data it generates right outcome from the labelled data. Thus, supervised learning is creating a mathematical model applying data that contains both the inputs and the required outputs.

For example, if we are provided with a basket filled with various types of fruits, the initial step is to train the machine one by one with different fruits:

- If the object is red in colour and is rounded and has curve at the top, then it is labelled as Apple.

- If the object is green, yellow in colour with shape as a long curvy cylinder it is labelled as Banana.

Now if the machine is asked to identify an arbitrary fruit from basket, then the machine uses the data learned from previous learnings and classify the specific fruit. Thus, it first uses the data to train itself and afterwards used it to test new data.

According to Goyal [16], machine learning techniques are used to address these three types of problem:

- Classification, that is, the evolution of a computer model by combining understandable labels with estimated value vectors. When a model is made from existing data 
(training data), it may be used to predict new scale labels.

- Regression, that is, the construction of a computer model based on training data by combining targeted continuous value or results with uninterrupted value input.

- Density estimation, that is, the limits of the delivery quantity to the power of metered data from samples without the effect or selected prices.

Unsupervised learning is the kind of machine learning that trains itself with the information that is neither labelled or classified and the algorithm acts on the information without direction. So mathematically it's creating an algorithm without any desired output and contains only inputs.

For instance, the analytics company segments the patients into smaller groups with similar disease to help the medical companies to reach them easily.

Unsupervised learning is divided into two kinds of algorithms:

- Clustering: For Grouping data

- Dimensionality reduction: For Compressing data

- Association: To describe the large populations of data i.e. If $X$ then $Y$

Reinforcement learning as the name suggests there is not any correct answer, and the reinforcement parameter tells us to perform the required task. So as there is no training data set and it learns from its experience. So, reinforcement learning is taking decisions sequentially. The output depends on current input and the next input depends on the output of previous input. Overall, supervised learning strategies applied to address the issues of segregation and reversal, and human measurement is done through an uneducated learning body. Supervised education is a body of knowledge that builds computer models / rules based on visual evidence. Fig. 1 depicts an overview on the types of machine learning techniques recently used. As implied by the name, supervised reading strategies train map activity from inputs to well-known extrudes in a randomized oriented reading, and then use those readings to predict output / labels for new invisible visuals. Unattended machine learning strategies are involved in determining the pattern or structure under a set of measurement data. Unlike supervised reading, unregulated learning algorithms do not require output or visual label.

\section{Fault diagnosis techniques using Artificial Intelligence}

The removal of critical aspects of damage is critical to the effective use of a machine learning machine damage test machine. However, it is not possible to define a large set of critical risk factors to approach and find the conditions of all types of structural systems (e.g., aerospace, public, oil, gas, etc.). and mechanical learning tools for such damage are critical features, thus providing students with a general guide to the type of discriminatory object and algorithm for classifying those features. We reviewed the skills of planning, integrating, and measuring Artificial neural network (ANN), support vector support (SVM), auto-regress models (AR models), $\mathrm{K}$ methods, and neighbours near $\mathrm{K}$ in a variety of critical aspects of damage in a controlled and uncontrolled manner in the following sections. Machine learning strategies developed from a combination of primarily learning algorithms are inspected under the "mixed" category, and the abovementioned machine learning strategies are described in the "others" section. As mentioned earlier, the main core concept of this review article is to provide critical damage reviews and machine learning algorithms for those features therefore, we shall not discuss the solid mathematical origins of learning algorithms.[16]

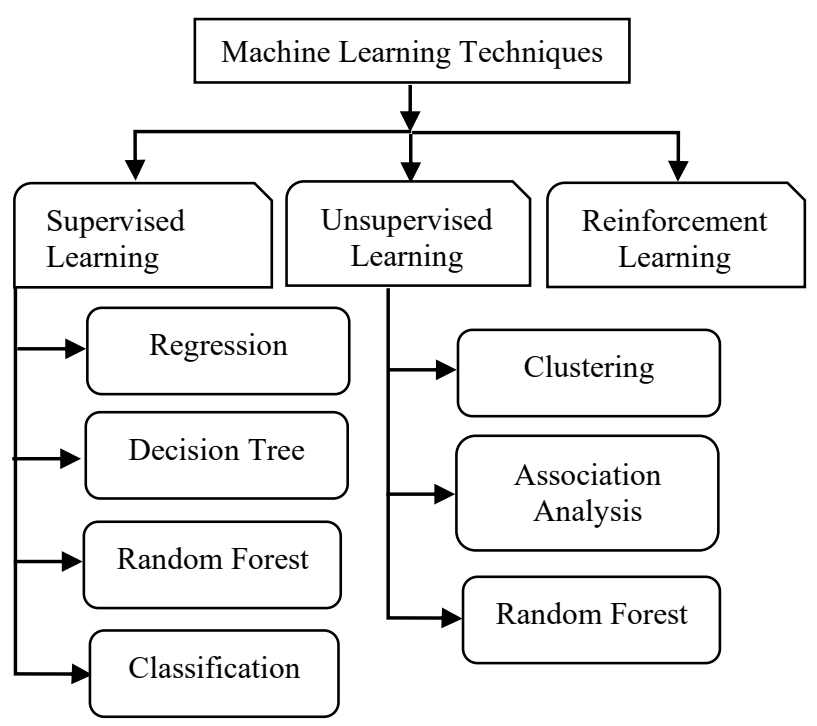

Fig. 1. Overview of Machine Learning

\subsection{Neural Network}

The history of the ANN can be followed back to the late 1800 's, when scientific attempts were attempted to duplicate the function of the human brain. The ANN are computing systems inspired from biological neuron as depicted in Fig. 2. Neural network can be either artificial or organic in nature. The advantage is we don't need to redesign the output criteria as the network gives the best result. The concept of neural network is gaining its importance in predicting fault diagnosis.
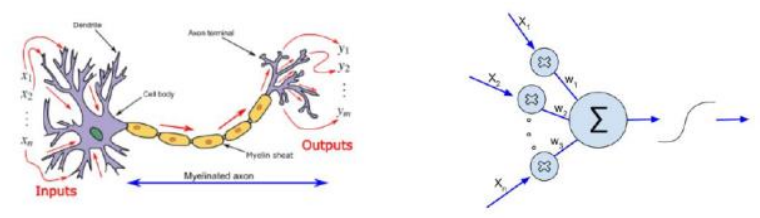

Fig. 2. Corelation of Biological Neuron with ANN

Neural networks can be categorised into different types. The common neural networks are described below:

In 1958, the oldest neural network perceptron was made by Frank Rosenbalt. It had a single neuron and was the simplest form.

Multilayer perceptron (MLP) or feedforward neural networks comprises an input layer and a hidden layer or 
several layers and an output layer. As most of the problem in world are nonlinear data is given to these models and used in computer vision, NLP (Natural language processing), NN (Neural Networks).

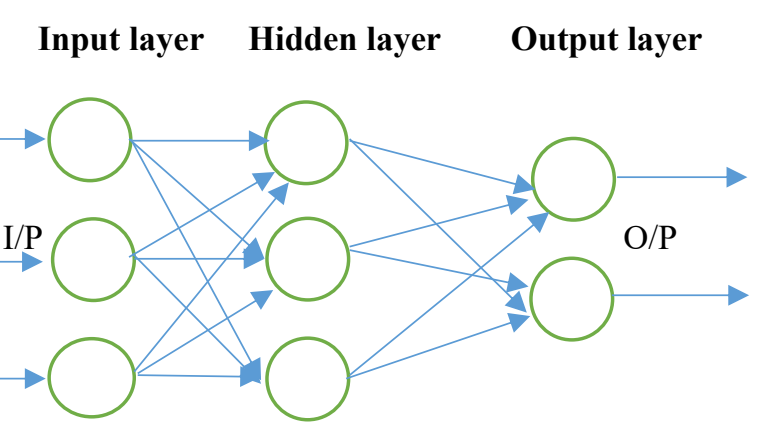

Fig. 3. Feed forward Neural Network

Fig. 3 shows a 2-layer neural network. In between the Input layer and Output layer there is the hidden layer with 3 neurons.

Convolutional neural networks (CNNs) are same as MLP and are specially used in image/pattern recognition and computer vision. To detect patterns, they use matrix multiplication and work on of linear algebra principles. Recurrent Neural Network (Fig. 4) use the time series data for prediction on future possibilities. They are known by their feedback loops.
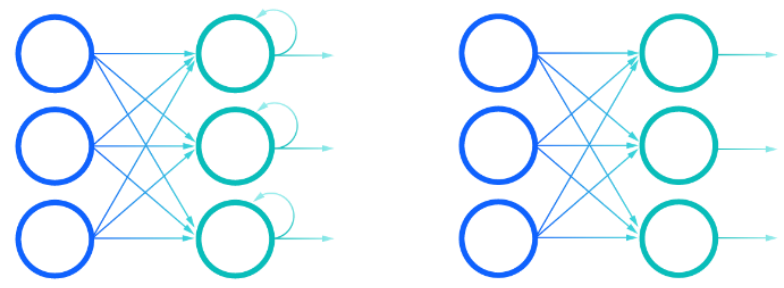

Fig. 4. Recurrent Neural Network vs MLP

Although, ANN was first hired to detect damage in 1991 by Gaboussi et al. [17]. At this stage, the ANN is inspected for damage assessment of the integrated structures. Islam and Craig [18] have used a neural-backspread network for segmentation and quantification of delamination in cleverly integrated laminates. The first five methods of the neural network were trained in the as traumatic factors, and the trained network was tested for invisible cases of delamination in various areas. Okafor et al. [19] used a backpropagation neural network to detect delamination size in a smart compound. Delamination sizes that do not match the size and the corresponding first four conditions have been used to train the neural network. The tested trained network with new delamination cases using the first four common wave test cases such as networking. The network successfully predicted an unbalanced average delamination size $(0.22$ and 0.82 ) but failed to predict an unlimited delamination size of less than 0.08. Sung et al. [20] Integrated neural network with Levenberg - Marquardt algorithm and general approach, finding the most accurate and reliable location for low-impact impact damage on intelligent integrated laminates. The neural network was trained, validated and tested during the arrival of acoustic waves generated by the impact on the PZT sensors as network input, and the location of the impact as the target output. For graphite / epoxy laminate $(330 \mathrm{~mm} \times 330 \mathrm{~mm} \times 3$ $\mathrm{mm})$, the proposed process predicted the impact area under a $5 \mathrm{~mm}$ error. Sammon et al. [21] merged x-ray diagnostics with a CNN (Convolution Neural Network), to diagnose and measure delamination in CRPF (Carbon Reinforced Polymer Composite). Every clip which are of $(201 \times 201$ pixels $)$ of the image has been assigned a class label according to the center pixel in the corresponding clip. The trained network allowed to identify and measure delamination of little sizes but was unable to estimate the magnitude of large delamination. Bar et al. [22] employed an Artificial neural network (ANN) to identify and differentiate the ineffective pathways in plastic-reinforced plastic parts from acoustic emission (AE) markers for the rigidity of the rigidity measurement measured with a film embedded in Polyvinylidene fluoride (PVDF). The Kohonen self-organizing feature map (KSOM) was hired to produce class data for various failure modes for instance Matrix crack, fiber-matrix debonding, fiber fracture). KSOM class data was applied for multilayer perceptron training (MLP). The proposed method has successfully discriminated against a variety of failures, regardless of the variance of the AE signal parameters associated with such methods. ANN was used to eliminate noise of several types from $\mathrm{AE}$ signals and to set $\mathrm{AE}$ events which are three different ways to fail in CFRP types by Bhat et al. [23]. The AE single attributes used as a descriptor were the time of ascent, calculation, strength, and height. It has been proved that KSOM and MLP are capable to effectively separate and classify noise of different sources from failure modes (fiber / matrix debond, fiber failure, matrix crack) of CFRP. Chetwynd et al. [24] monitored a multilayer perceptron neural network for planning and deferral detection problems on a reinforced carbon fibered panel with transducers attached to the surface. Much damage was done to the area through the power applicant, and the Lamb's response was obtained in healthy and damaged cases. The Lamb's wave response in each case was transformed into an indicator of scale art by external analysis. New indicators of 28 sensory pathways (ignoring modified pathways) of the eight face-lift sensors have been used to incorporate MLP segments and retrospective networks. The MLP separation network was applied for the separation of damage and non-damaged areas of the panel, and the MLP recovery network was applied to measure the position of the damage to the panel.

\subsection{Support vector machine (SVM)}

Support Vector Machine (SVM) places the indivisible feature space in the high magnitude space as a result of a kernel function, just as a separate line hyperplane can be built in a space of high magnitude. Details of SVM mathematics can be found at Reference [25]. In theory, SVM has been applied for erroneous mechanical diagnostics [26], gear error diagnostics under dynamic condition [27], local source for acoustic emission [28], 
and diagnostic feature of turbine engine [29,30], etc. Das et al. [31] used single phase vector mechanics (SVMs) to categorise and identify the four categories (notch, saw cut, delamination, hollow holes) of damage to intelligent integrated laminates. A frequency-time based method (frequency (Gabor's spectrogram process) and time embedding process (tap-delay method) was used to exclude critical factors in piezoelectric sensory responses. The results of the classification of SVMs in the same class have shown that the embedded features are better than the time-often features of precision. Prashant and Sung [32] developed SVM-based approach to detecting damages online on a helicopter rotor blade. Vibration harness loads and heavy door times under helicopter assembly were used as destructive features of matrix cracks and debonding / delamination injuries. The critical factors of damage were divided into three categories (matrix cracking to saturation, delamination initiation, and excessive delamination). SVM. Farooq et al. [33] found that the effectiveness of SVM and ANN testing and the intensity of fracture damage in a fiber-reinforced panel. The Static strain mixed with Gaussian noise was used for the cross validating and training of SVM and ANN. SVM was found to be more effective than ANN for damage detection and magnification tests. Dib et al. [34] has established a novelty classifier based on a single SVM class for detection of damage. The performance of the classifier was tested on data from the impact damage test on the glass fiber composite plate. By detecting discriminatory features, the Lamb wave was split into Lbins and the Fourier transformation was calculated in each time bin. The feature vector is formed from the amplitude and signal phase of each time of the bin.

\subsection{Auto regressive models (AR models)}

The Auto-regression (AR) model a mathematical tool used to describe time-varying processes by predicting the different interest based on previous datas of the same variability and parameters of the AR model. Other relevant instances of AR models for damage detection are error detection and compression monitoring [35], airconditioned injury detection [36], damage detection of frame structures [37], and structural health monitoring of public infrastructure [38][39]. Nardi et al. [40] described the detection of low-velocity delamination with low impact on smart composite laminates with auto-regression (AR) models. The correct order of the AR model was identified to compare the damaged data/undamaged data, and the compatible AR parameters were transformed into a new small space by linear discrimination analysis (LDA).

Intermediate phase $\left(S_{w}\right)$ and intermediate (S) phase matrices are defined by the changed AR parameters used to describe the new formed matrix $\mathrm{V}\left(V=S_{w}^{-1} \times S_{b}\right)$. The first three eigenvectors and the compatible eigenvalues of the matrix $\mathrm{V}$ were selected as critical features. Fig. 5 shows the clusters of the various damaged and untreated cases V-shaped based on the proposed procedure. Here, the harmless regions of the three plates are represented by $\mathrm{C} 1_{U}$ (asterisk), $\mathrm{C} 2_{U}$ (square) and $\mathrm{C} 3_{U}$ (diamond), while the damaged areas of the same plates are given by $\mathrm{C}_{D}$ (cross), $\mathrm{C} 2_{D}$ (circle) and $\mathrm{C} 3_{D}$ (triangle). $\mathrm{C} 1_{D}, \mathrm{C} 2_{D}$ and $\mathrm{C} 3_{D}$, respectively, fell under the power of $20 \mathrm{~J}, 8 \mathrm{~J}$ and 12 J. Vamvoudakis-Stefanou et al. [41] compared the performance of two non-parametric methods [i.e., spectral density force (PSA) and transmittance (TF) function) and one parametric method [autoregressive model (AR)], to determine the impact disturbance in composite signals. All the three methods were trained with the signals of a arbitrary vibration response of a healthy pole. The efficacy of the trained damage detection models was tested by examining those with random vibration responses from 22 healthy and 3 damaged tumours. It was observed that among the three methods of detection of damage, the parametric method of AR is effective and potent.

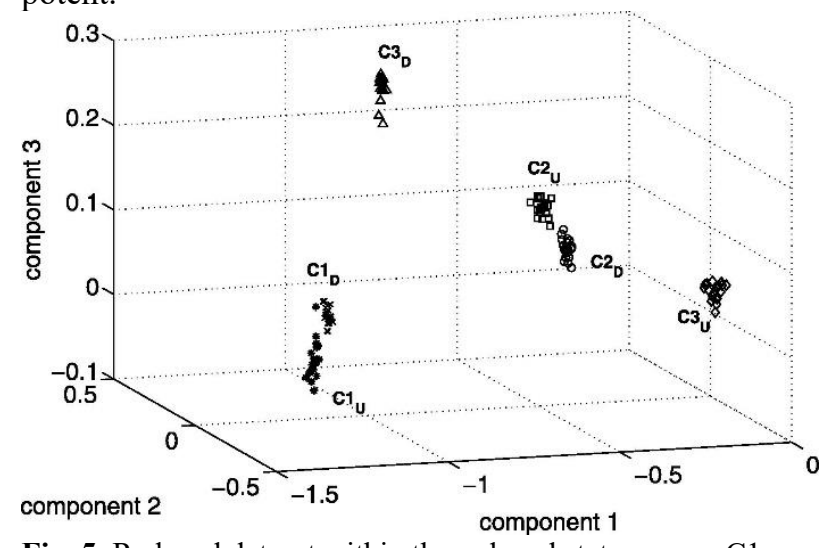

Fig. 5. Reduced dataset within the reduced state space - $\mathrm{C} 1 \mathrm{U}$ (asterisk), $\mathrm{C} 1_{\mathrm{D}}$ (cross), $\mathrm{C} 2 \mathrm{U}$ (square), $\mathrm{C} 2 \mathrm{D}$ (circle), $\mathrm{C} 3_{\mathrm{U}}$ (diamond), $\mathrm{C} 3{ }_{\mathrm{D}}$ (triangle).AR model order $\mathrm{p}=25$ [40]

Brincker et al. (1995) [42] used mathematical analysis based on natural wave shifts to detect damage of two concrete beams with varying reinforcement ratios. The modal parameters (natural waves and modes reduction) were calculated from a series of response time (acceleration) using an automatic measurement of automatic motion (ARMA), similar to that used by Blincker et al. (1995) [42] in place of the beach platform. In reference to Davies and Hammond (1984) [43], Pandit and Mehta (1985) [44] and Safak (1991) [45], Brincker et al. (1995) [42] states that ARMA models was developed primarily for economic use and engineering (electrical), but as they are the most effective way to predict modal parameter than the Fast Fourier Transform (FFT), their use in building systems has been growing presently. A more updated version is the ARMA combined with exogenous input (ARMAX). The ARMAX $[n, m]$ order model that puts the time response $\mathrm{y}(t)$ as one linear combination of the last $n$ responses and also the last $\mathrm{m}$ unknown inputs and $\mathrm{p}$ known inputs is described by:

$y(t)=\sum_{i=1}^{n} c(i) y(t-i)+\sum_{i=1}^{n} b(i) x(t-i)-\sum_{i=1}^{m} d(i) e(t-i)+e(t)$

$\mathrm{c}(i)=$ auto-regressive parameter which expresses as a linear regression the response $\mathrm{y}(t)$ of the last ' $n$ ' values $\mathrm{d}(i) \& \mathrm{~b}(i)=$ moving average parameters which expresses as a linear regression the response $\mathrm{y}(t)$ of the last values of, sequentially, an undetermined input time series.

$\mathrm{e}(t)=$ believed as white noise, and a determined time series, $\mathrm{x}(t)$. 
The parameters $\mathrm{c}(i)(A R), \mathrm{d}(i)(M A)$ and $\mathrm{b}(i)(\mathrm{X})$ are determined by reducing the variance of $\mathrm{e}(t)$, the noise time series i.e., based on the determined response and on the forecasted response given by eqn. (1). Thereafter, AR parameters are calculated and the roots of the characteristic polynomial equation which detain these parameters can be associated to the natural frequencies and modal damping. So, to examine these parameters and variations with time the ARMAX can be used. If they provide a balanced measure of structural changes, it could be also described a statistically significant indicator that looks at differences in natural waves. It has been expressed that value indicators are delicate to damage to the building but are unable to provide a calculation of the damage area. The difference between ARMA and ARMAX was very small which was found finally, with the study parameters. Therefore, it was justified that knowing of the input signal is not necessary for detecting damage to the studied columns. To evaluate the internal delamination of multi-layer plates, nonlinear ARMA was combined with exogenous input models (NARMAX) by Wei et al. (2005) [46]. They declare that the corelation between input and output does not exist in the line of integrated multilayer panels. The NARMAX model which was proposed by Leontaritis and Billings (1985) [47] according to them it was used as a standard parametric form for modelling systems without lines. NARMAX models define non-linear systems based on different ratings relating to current output in the combination of inputs and older results. To measure the model parameters of damaged vibrational systems the design of the suggested model is used, and the internal observations are gathered by combining the model parameters of solid and damaged systems. The results showed that, in the case study, NARMAX models permits for the behavioural investigation of composite models and the detection of damage (delamination in particular) in composites. Centred around the concept of a dynamic coherent system Lu and Gao (2005) [48] presented a design as an automatic predictor model along exogenous input (ARX). Later on, simplification, the model was presented in such a way that only the response signals were affected, and the response in one place is selected as the input model. The established model's residual error, when used as the standardized signals itself, indicates a structural change, and critical factor of damage was found with the general deviation of the residual error. It was noted that the proposed ARX model may behave inappropriately when the selected input response area is close to the damage site. Two different procedures are performed using two different input locations they recommend that in the diagnostic case for this reason. It was also observed that further research is needed as the general deviation of the residual error of the ARX model, although delicate to the existence of damage and does not provide an exact indication of the extent of the damage, so. In their earlier work, Sohn and Farrar in 2001[15] introduced, AR-ARX the two-iteration model to forecast the time series and to apply later the standard deviation of the remaining error deviation as a feature of damage sensitivity. The difference between actual ratings and predicted responses from a mix of AR and ARX models is a fatal error.

\subsection{Principal component analysis (PCA)}

The Principal Component Analysis (PCA) is used immensely to minimise the intensity of critical risk factors or signals of strong structural responses in general $[49,50]$. In some cases, the reduction in size allows for a closer look at the clusters of critical risk factors and gives appealing results in detecting and categorising of damage [51]. Other PCA programs are of sensor validation [52], and differentiating the environmental consequences from structural damage, etc. With the aim of discriminating the acoustic signatures of damage methods in glass / polyester composites. Fuzzy C Means (FCM) integration with the primary investigation of acoustic emission data collection was proposed by Oskouei et al. [53]. The aim was to discriminate the acoustic signatures of damage methods used in glass or polymer composites. Awakening time, calculation, strength, height, high frequency and length of $\mathrm{AE}$ signals were applied as definitions of fiber-matrix debonding, matrix fragmentation, and fiber failure.

\subsection{K-means \& K-nearest neighbours}

$\mathrm{K}$-means is a contumacious learning method that detects $\mathrm{K}$-groups or K-clusters within un-labelled data. The number of groups or groups are described by the name K. Every point of data in the data is set to a targeted group by the $\mathrm{K}-$ means algorithm, based on the point distance from the centre of a given group. K-Nearest Neighbours $(\mathrm{KNN})$, on the other hand, is a supervised learning method often used for integration and classification. ' $\mathrm{K}$ ' at KNN implies the number of adjacent neighbours applied to separate the test sample. For the integration of acoustic discharge signals from a solidarity test of unidirectional glass / polyester composite the components were monitored and unchecked by Godin et al. [54]. AE signals are initially matched by k-means based on life expectancy, calculation value, duration, magnitude, and count to the top of the AE signals. Collections identified by $\mathrm{k}$ methods have been used as data labelled by the supervised division of KNN. For the division of new data, the trained KNN separator was realized. The Kohonen map's unsupervised segmentation showed similar results to the segmentation of the KNN. The proposed method was successful and divided the AE signal linked with matrix fragmentation and interfacial decohesion. Combination of k-means algorithm with genetic algorithm for the clustering of several damage methods in test mode I delamination of sandwich assembly. Fig. 6 shows the process used for unsupervised damage planning. Here, the I delamination mode test is performed on the sandwich case and its failure events (fiber breakdown, matrix cracking, and major damage) are taken with the AE sensor. Discrimination characteristics of AE symptoms identified by each failure mode and key analysis (PCA) are hired to reduce the size of the discrimination feature space. Reduced features are grouped together by different combinations with a combination of a genetic algorithm and a k-means 
algorithm. The identified clusters are tested with a different damage process.

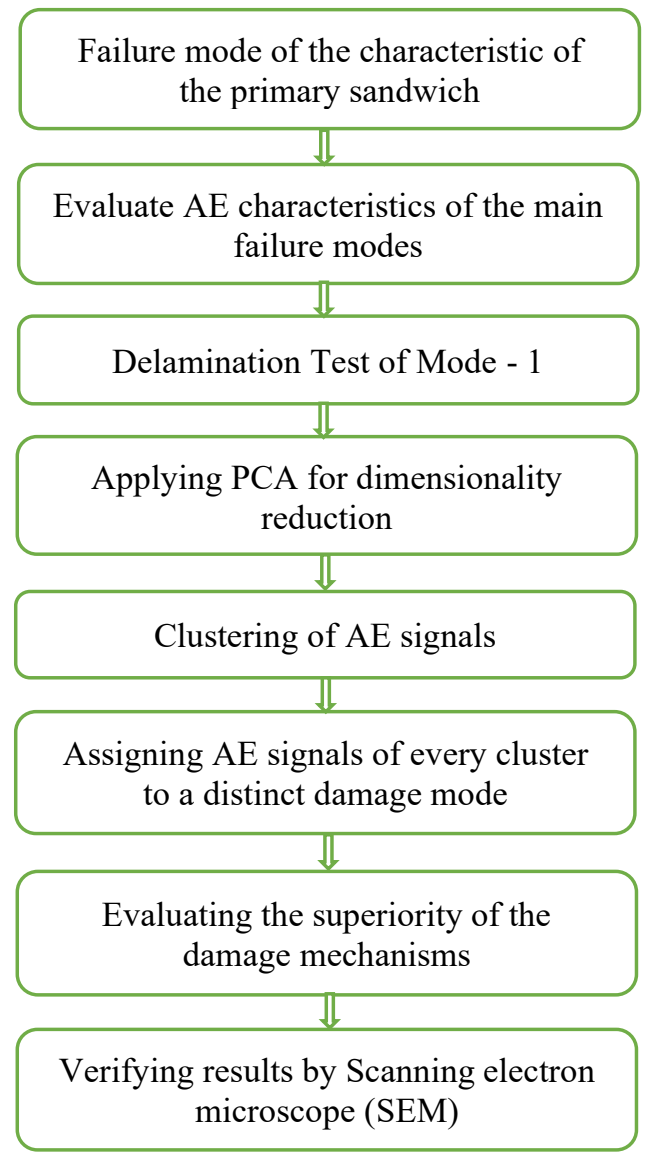

Fig. 6. Damage (in laminated composite structure) classification procedure [6]

\section{Additional Techniques}

There are several types of algorithms based on Artificial Intelligence techniques for fault diagnosis and have been analysed and presented in this section. The methods can be widely divided into three categories as follows:

I. Fuzzy Inference technique

II. Genetic algorithm (GA) technique

III. Hybrid technique

\subsection{Fuzzy inference technique}

Hoshyar.et.al. [55] in 2019 suggested how to detect and visualize damage based on multiple non-destructive testing methods (NDT). An ML-based learning method (MV) based system is developed to prevent misinterpretation of the event. The aim is to identify cracks early on, to reduce the risk of failure in the structures. Theoretical analysis and evaluation are taken by inserting performance indicators into smart aggregate (SA) data with a base of concrete and reinforced steel (RC). Images were processed using various image processing algorithms. The algorithms were based on Fourier, Wavelet transform, Fuzzy c-mean, Gaussian filter, Median filter, and Gradient size. Proposed algorithms have made it easier to clearly identify adverse events in the buildings to be performed. The results prove that algorithms have the potential to effectively enhance microwave imaging with poor resolution, and to identify areas of interest, especially in systematic health surveillance. Comparing different methods of artificial intelligence for fault diagnostic error, vibration signals collected from faulty composite plates by Moghaddam et al. [56] in 2019. Using varieties of time-frequency signal analysis methods and processing methods, many features were extracted from these indicators and the most effective features containing additional details these composite plates were presented to different classification systems. The faults in the composite plates were shown by the classification systems. The different types of segregation systems used in this study are adaptive neurofuzzy inference system (ANFIS), Support Vector Machine (SVM), K-Nearest Neighbour (KNN), Artificial Neural Networks (ANN). Also, the Extended Classifier System (XCS) algorithm, as well as the proposed XCS algorithm was discussed. The results of the study showed ANFIS has higher levels of accuracy, while this method has a very high process time with same number of repetitions. The generalised ANFIS modules are designated schematically in Fig. 7.

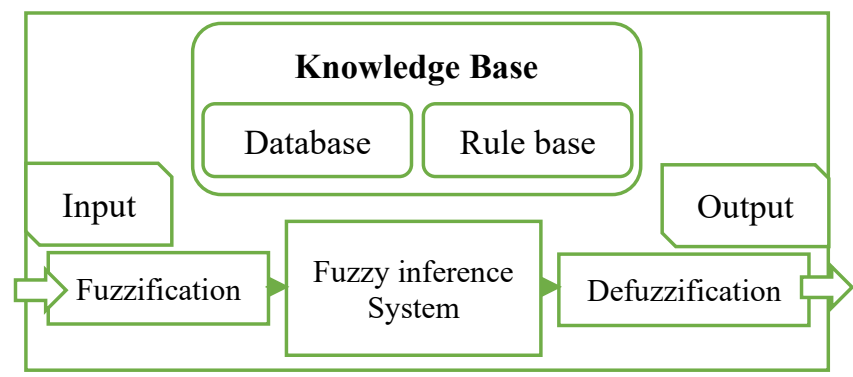

Fig. 7. Generalised ANFIS module

The precision of the proposed XCS method was lower than that of ANFIS, still the duration of the process was smaller than the ANFIS method with the equal number of repetitions. It is recommended that the proposed method could be used to resolve this problem as the time needed for computer performance and the percentage of accuracy in engineering problems such as the detection of defects in composites is acceptable. Using displacement and forced based deviations between damaged and undamaged conditions in 2007 Pawar.et.al [57] introduced a Genetic Fuzzy algorithm for online prediction of physical damage and life consumptions. In addition, an online forecast for physical damage and useful life was made using strains measured along the Rotor head length. It is also evident that the success rate of complex genetic systems depends on the number of experiments, the type of experiments and training, and the level of test noise. The genetic systems work well with noisy data and are recommended for online check-up of composites rotor blades.

To predict the damage characteristics a composite cantilever beam which was subjected to vibration a fuzzy based model using triangular, trapezoidal and Gaussian membership functions was developed by Agarwalla in 2018 [58]. The relative damage position and relative damage severity was calculated. The first three natural frequencies and the first three-mode shape variation 
which is the input required for the fuzzy-based model are determined by the Finite Element Analysis of the damaged cantilever beam exposed to natural vibrations. It has been observed from the comparison of the results obtained from the proposed model and the evaluation that the deviation of the errors is within the acceptable limit of the Fuzzy Gaussian model to that of the fuzzy trapezoidal and fuzzy triangular model. Therefore, the identification of composite damage was done with the proposed tool using the Fuzzy Gaussian model and was effective. Kim et al. [59] in 2007 introduced a computer-based fault diagnosis for concrete structures using Fuzzy set theory. They used crack indications and identities of damage in the constructing the rooms for the proposed fuzzy system. While using the advanced method of detecting crashes the proposed system provided results like those obtained by the genuine system.

\subsection{Genetic algorithm (GA) technique}

Based on Weibull's ability to calculate damage to the interface and Cox model to evaluate and set different values used in the simulation model a method was given by in 2020 by Belkheir.et.al [60] based on genetic algorithm. The results were confirmed by the degree of damage to the interface found in the two studies and that the damage to the interface Basalt / Polyester composite/ Carbon high modulus (HM) was significantly lower by $13 \%$ compared to that of Basalt /Polyester/ Carbon high strength (HS). From the calculation it was found that the results were in accordance with the cox analytical results. Young's Modulus have a significant impact on the shear strength of the fiber / matrix interface of composite material. As discussed earlier in which Zhongqing Su.et.al 2005 [8] compared the effectiveness of artificial neural networks (ANNs) and genetic algorithms (GAs) in a delamination test on glass fiber reinforced epoxy composite laminates. With GA-based identification, a conceptual model with a vibrating work was developed to link the boundaries of delamination with the changing eigenvalues of a building. The responses of selected GF / EP laminate beams having several delamination were taken applying embedded fiber Bragg grating sensors as a proven method. From which structural eigenvalues were extracted and used inconsistently to conduct damage tests with GA and ANN. From the results it was found that more effort is required to prepare for ANN training, compared to GA implementation.

\subsection{Hybrid technique}

Fuzzy thinking, neural network, and genetic algorithm have been integrated into the process of developing crack/fault diagnostic methods. Xiaoma and Qingzhen [61] in 2010 proposed a robust method based on the hybrid algorithm i.e. Radial Basis Function Neural Network (RBFNN) to diagnose composite damage. Using a wavelet series, the signal features were extracted and input to a hybrid algorithm RBFNN for network training and damage detection. Artificial neural networks have been studied and used in many fields because of their nonlinear mapping ability and real-time calculation ability. It has excellent previous use to use ANN to detect compounded items. In this paper, it was learned to use RBFNN to obtain compound identification. The network has some important features - simple arithmetic, fast learning ability, and dynamic performance capabilities, for example. Modelling of composite materials is avoided. Therefore, the damage detection method based on the RBFNN hybrid algorithm is a useful diagnostic method. The research results of this paper suggest that this approach may point to the errors of the composite material. Jena [62] in 2018 made an artificial intelligence (AI) process used to eliminate the error diagnosis in the FRC beam. By the way, the neuro fuzzy hybrid has been used as a modified AI process. The results gathered from the experimental study and the direction of the woven fibre are applied as input to the neural network (NNT) process to determine the location of the fracture and the depth of the fracture as output. Matching the output with the expected results obtained, by inserting inputs into the fuzzy logic system from both NNT and experiment methods, so the task is to achieve more precise results. Continuously extracted from NNT and the results obtained in the test were combined and considered as input to the logic system (FLS) for the solution of getting more accurate results. The conclusion in the current study is that fiber orientation works best for FRC beam solidity and a neuro-fuzzy hybrid technique that produces more specific results. As this regression method is a nondestructive method that can be used as a continuous form to detect an error in the FRC beam. Alazzawi and Wang [63] in 2021 proposed a network-based algorithm and a hybrid training method aimed to detect, measure and build structural moisture. The present method was tested experimentally, many structured and undamaged structural conditions were performed, obtained signal samples of the PZT impedance response of the time. As the results show, the method has achieved significant performance in detecting damage, testing the magnitude of the damage and damaging local recognition with high accuracy and reliability.

\section{Conclusion:}

With the increase in use of composite materials in different sectors the need for early defect assessment is the key factor. In this present paper various crack/fault analysis methods mostly based up on the dynamic response of the advanced material structure have been highlighted. Focus is given on the types of damage sensitive features with quite a few kinds of damage in composite structure. There are several other techniques namely finite elements method (FEM) and wavelet technique to detect the crack/fault. However, emphasis is given on fuzzy logic and AI techniques and Machine learning methods such as ANN, GA implemented in past to assess fault in laminated composite structure. Both supervised and unsupervised machine learning techniques have been reviewed. These methods proved to be efficient for estimating crack locations and their intensity during the study of vibration signatures. With the exposure in machine learning in various areas has allowed the 
research community to put interest in it, specifically to facilitate the resource management fields, economics and safety. The summary of the currently reviewed articles is outlined in the following lines.

1. To build the predictive models unsupervised learning can be used to obtain the class knowledge for several types of failure with the labelled data.

2. The lowest and highest frequency information of acoustic emission signals represent the fiber breakage and cracking of matrix respectively while in frequency range of fiber breakage and matrix cracking represent debonding.

3. Fuzzy controller was developed using Fuzzy logic taking the data from operated machines. Several types of fuzzy function such as triangular, Gaussian were used for fault detection and to constitute Fuzzy inference system.

4. An inverse diagnostic tool was developed using Genetic Algorithm tool. The calculation time for fault detection using GA was in milliseconds.

5. Scientists presented various hybrid techniques, like GA fuzzy, GA fuzzy neural, GA neural network for damage detection by predicting the crack parameters.

Generally, the supervised machine learning algorithms were less expensive computationally and depend on low end machines like CPUs which perform good on less learning data and need features from the data (sources). However, unsupervised learning are expensive and depend on high end machines like GPUs and need huge amount of training data and find discriminative features based on raw loaded data.

\section{References}

1. A. Macke, B. F. Schultz, P. K. Rohatgi, N. Gupta, Metal Matrix Composites for Automotive Applications (2013)

2. R. A. Smith, Mater. Sci. Eng. III, 103 (2009)

3. A. Raghavan, M. Balasubramanian, H. S. Kim, Comput. Struct. 161, 2603 (2006)

4. J. W. Sohn, H. S. Kim, Int. J. Precis. Eng. Manuf. 16, 597 (2015)

5. A. Khan and H. S. Kim, Sensors (Switzerland) 19, (2019)

6. A. Khan, H. S. Kim, and B. D. Youn, Int. J. Mech. Sci. 26,131-132 (2017)

7. A. Raghavan, Diss. Abstr. Int. Vol. 68-11, Sect. B, Page 7465.; Adviser Carlos E. Cesn 975, 91 (2007)

8. Z. Su, L. Ye, Y. Lu, J. Sound Vib. 295, 753 (2006)

9. A. Yaghoobi and M. G. Chorzepa, Comput. Struct. 161, 43 (2015)

10. V. Giurgiutiu, Struct. Heal. Monit. with Piezoelectric Wafer Act. Sensors 1 (2014)

11. J. J. Valletta, C. Torney, M. Kings, A. Thornton, J. Madden, Anim. Behav. 124, 203 (2017)
12. D. Kateris, D. Moshou, X. E. Pantazi, I. Gravalos, N. Sawalhi, S. Loutridis, J. Mech. Sci. Technol. 28, 61 (2014)

13. S. Park, J.-J. Lee, C. Yun, D. J. Inman, J. Mech. Sci. Technol. 21, 896 (2007)

14. Wang H., Ma C., Zhou L., Proc. - Int. Conf. Inf. Eng. Comput. Sci. ICIECS (2009)

15. H. Sohn, C. R. Farrar, F. Hemez, J. Czarnecki, 1 (2001)

16. K. Worden, C. R. Farrar, G. Manson, and G. Park, Proc. R. Soc. A Math. Phys. Eng. Sci. 463, 1639 (2007)

17. X. W. J. Ghaboussi,J. H. Garrett Jr., J. Eng. Mech. 117, 132 (1991)

18. A. S. Islam and K. C. Craig, Smart Mater. Struct. 3, 318 (1994)

19. A. C. Okafor, K. Chandrashekhara, Y. P. Jiang, Smart Mater. Struct. 5, 338 (1996)

20. D. U. Sung, J. H. Oh, C. G. Kim, C. S. Hong, J. Intell. Mater. Syst. Struct. 11, 180 (2000)

21. D. Sammons, W. P. Winfree, E. Burke, S. Ji, AIP Conf. Proc. 1706, (2016)

22. H. N. Bar, M. R. Bhat, and C. R. L. Murthy, Compos. Struct. 65, 231 (2004)

23. C. Bhat, M. R. Bhat, C. R. L. Murthy, Compos. Struct. 61, 213 (2003)

24. D. Chetwynd, F. Mustapha, K. Worden, J. A. Rongong, S. G. Pierce, J. M. Dulieu-barton, Strain 44, 298 (2008)

25. H. Z. Huang, H. K. Wang, Y. F. Li, L. Zhang, Z. Liu, J. Mech. Sci. Technol. 29, 151 (2015)

26. G. M. Lim, D. M. Bae, J. H. Kim, J. Mech. Sci. Technol. 28, 2947 (2014)

27. Z. Xing, J. Qu, Y. Chai, Q. Tang, Y. Zhou, J. Mech. Sci. Technol. 31, 545 (2017)

28. Y.-S. L. Kwang-Ro Kim, J. Mech. Sci. Technol. 28 (8), (2014)

29. Y. Kim, J. Jang, W. Kim, T. S. Roh, D. W. Choi, J. Mech. Sci. Technol. 26, 1623 (2012)

30. D. H. Seo, T. S. Roh, D. W. Choi, J. Mech. Sci. Technol. 23, 677 (2009)

31. S. Das, A. Chattopadhyay, A. N. Srivastava, AIAA J. 48, 705 (2010)

32. P. M. Pawar and S. N. Jung, J. Intell. Mater. Syst. Struct. 19, 1217 (2008)

33. M. Farooq, H. Zheng, A. Nagabhushana, S. Roy, S. Burkett, M. Barkey, S. Kotru, and E. Sazonov, Smart Sens. Phenomena, Technol. Networks, Syst. Integr. 2012 8346, 83461O (2012)

34. L. U. G. Dib, O. Karpenko, E. Koricho, A. Khomenko, M. Haq, Mater. Today Proc. 27, 0 (2019)

35. F. Cong, J. Chen, G. Dong, J. Mech. Sci. Technol. 26, 301 (2012) 
36. L. D. Avendaño-Valencia and S. D. Fassois, J. Phys. Conf. Ser. 628, (2015)

37. Z. Wang, K. C. G. Ong, Eng. Struct. 31, 1265 (2009)

38. E. Peter Carden, J. M. W. Brownjohn, Mech. Syst. Signal Process. 22, 295 (2008)

39. K. K. Nair, A. S. Kiremidjian, K. H. Law, J. Sound Vib. 291, 349 (2006)

40. D. Nardi, L. Lampani, M. Pasquali, P. Gaudenzi, Compos. Struct. 151, 108 (2016)

41. K. J. Vamvoudakis-Stefanou, J. S. Sakellariou, S. D. Fassois, Proc. ISMA 2014 - Int. Conf. Noise Vib. Eng. USD 2014 - Int. Conf. Uncertain. Struct. Dyn. 3839 (2014)

42. M. E. u. Brincker, R. Andersen, P. Kirkegaard, P.Henning, Ulfkjær, J. P.Brincker, Rune Kirkegaard, Poul Henning; Andersen, P. Martinez, (1994)

43. P. Davies and J. K. Hammond, J. Vib. Acoust. Trans. ASME 106, 40 (1984)

44. S. M. Pandit and N. P. Mehta, J. Sound Vib. 122, 413 (1988)

45. E. Şafak, J. Struct. Eng. 117, 3064 (1991)

46. Z. Wei, L. H. Yam, and L. Cheng, Compos. Struct. 68, 109 (2005)

47. I. J. Leontaritis and S. A. Billings, Int. J. Control 41, $303(1985)$

48. Y. Lu and F. Gao, J. Sound Vib. 283, 1031 (2005)

49. G. Manson, K. Worden, K. Holford, R. Pullin, J. Intell. Mater. Syst. Struct. 12, 529 (2001)

50. L. E. Mujica, J. Vehí, M. Ruiz, M. Verleysen, W. Staszewski, K. Worden, Mech. Syst. Signal Process. 22, 155 (2008)

51. B. Huang, B. H. Koh, H. S. Kim, Comput. Struct. 141, 26 (2014)

52. G. Kerschen, P. De Boe, J. C. Golinval, K. Worden, Smart Mater. Struct. 14, 36 (2005)

53. A. Refahi Oskouei, H. Heidary, M. Ahmadi, M. Farajpur, Mater. Des. 37, 416 (2012)

54. N. Godin, S. Huguet, R. Gaertner, L. Salmon, NDT E Int. 37, 253 (2004)

55. A. N. Hoshyar, M. Rashidi, R. Liyanapathirana, B. Samali, Appl. Sci. 9, (2019)

56. R. K. Moghaddam ,N. M. Yazdan, Mech. Eng. 23, 113 (2019)

57. P. M. Pawar, R. Ganguli, Mech. Syst. Signal Process. 21, 2212 (2007)

58. D. K. Agarwalla, Int. J. Data Sci. 3, 170 (2018)

59. Y. M. Kim, C. K. Kim, G. H. Hong, Comput. Struct. 85, 1828 (2007)

60. A. B. Belkheir Mohammed, Bendouma Doumi, Allel Mokaddem, Researchgate (2020)

61. D. Xiaoma, S. Qingzhen, ICCET 2010 - 2010 Int. Conf. Comput. Eng. Technol. Proc. 1, 492 (2010)

62. P. C. Jena, Mater. Today Proc. 5, 19216 (2018)
63. W. O. Alazzawi, D. Ansheng, J. Mech. Sci. Technol. (2021) 